\title{
Damage- and pathogen-associated molecular patterns play differential roles in late mortality after critical illness
}

\author{
John Eppensteiner, ${ }^{1,2,3}$ Jean Kwun, ${ }^{1}$ Uwe Scheuermann, ${ }^{1}$ Andrew Barbas, ${ }^{1}$ Alexander T. Limkakeng, ${ }^{1,2,3}$ \\ Maggie Kuchibhatla, ${ }^{4}$ Eric A. Elster, ${ }^{3,5}$ Allan D. Kirk, ${ }^{1,3}$ and Jaewoo Lee ${ }^{1}$ \\ 'Department of Surgery and ${ }^{2}$ Division of Emergency Medicine, Duke University, Durham, North Carolina, USA. ${ }^{3}$ Surgical \\ Critical Care Initiative (SC2i), Bethesda, Maryland, USA. ${ }^{4}$ Department of Biostatistics and Bioinformatics, Duke University, \\ Durham, North Carolina, USA. ${ }^{5}$ Department of Surgery, Uniformed Services University of Health Sciences and Walter Reed \\ National Military Medical Center, Bethesda, Maryland, USA.
}

Multiple organ failure (MOF) is the leading cause of late mortality and morbidity in patients who are admitted to intensive care units (ICUs). However, there is an epidemiologic discrepancy in the mechanism of underlying immunologic derangement dependent on etiology between sepsis and trauma patients in MOF. We hypothesized that damage-associated molecular patterns (DAMPs) and pathogen-associated molecular patterns (PAMPs), while both involved in the development of MOF, contribute differently to the systemic innate immune derangement and coagulopathic changes. We found that DAMPs not only produce weaker innate immune activation than counterpart PAMPs, but also induce less TLR signal desensitization, contribute to less innate immune cell death, and propagate more robust systemic coagulopathic effects than PAMPs. This differential contribution to MOF provides further insight into the contributing factors to late mortality in critically ill trauma and sepsis patients. These findings will help to better prognosticate patients at risk of MOF and may provide future therapeutic molecular targets in this disease process.

Conflict of interest: The authors have declared that no conflict of interest exists.

Copyright: () 2019, American Society for Clinical Investigation.

Submitted: February 14, 2019

Accepted: July 26, 2019

Published: August 22, 2019

Reference information: /CI Insight. 2019;4(16):e127925.

https://doi.org/10.1172/jci.

insight.127925.

\section{Introduction}

Multiple organ failure (MOF) is the leading cause of late mortality and morbidity in patients who are admitted to intensive care units (ICUs) $(1,2)$. Systemic inflammatory response syndrome (SIRS) that follows significant innate immune stimulatory events, such as severe injury and infection, results in MOF and subsequent death. Although late complications in trauma and sepsis patients begin with innate immune stimulation, there remains a discrepancy in the incidence of MOF and late mortality in the 2 disease states. MOF incidence and mortality in sepsis patients reaches much higher rates than those of patients hospitalized after severe trauma (3-5).

The dysregulated immune response that leads to late complications in both sterile and septic insults has been well-studied, yet pathogenesis is still incompletely understood. There exists a complex pathophysiologic interplay of innate immune stimulators, such as damage-associated molecular patterns (DAMPs) and pathogen-associated molecular patterns (PAMPs), in the development of MOF and mortality in trauma and sepsis patients (6-10). Both DAMPs and PAMPs trigger inflammatory responses through innate immune receptors, such as TLRs (11). Microbial infection leads to robust inflammation via TLR downstream signaling pathways, such as the NF- $\mathrm{B}$ pathway (12). This PAMP-mediated and TLR-driven process results in the canonical cytokine storm and subsequent end-organ damage commonly implicated in the systemic pathogenesis of sepsis (13). Alternatively, sterile insults are propagated mainly by DAMPs via the same TLRs, leading to systemic organ damage.

Hypercoagulability is another risk factor for late mortality in sepsis and trauma patients. Early disseminated intravascular coagulation (DIC) and sustained SIRS were demonstrated as strong determinants for post-trauma MOF and late mortality (14). Early trauma-induced DIC, organ damage, and nosocomial infection-induced DIC cooperatively led to continuous DIC that is an independent risk factor for MOF incidence and late mortality in trauma patients (15). Interestingly, coagulation parameters and the clinical course of DIC was significantly different between sepsis and trauma patients (16). Certain types of DAMPs 
(e.g., high mobility group box 1 [HMGB1] and extracellular nucleic acids) and bacterial inorganic phosphate are known as potent procoagulants $(17,18)$.

Thus, we hypothesized that there is a difference in DAMP- and PAMP-driven innate immune stimulation and coagulation activation, which accounts for the variance in pathogenesis of MOF and late mortality in trauma and sepsis. This difference is the result of several contributing factors that we have demonstrated in this study. Notably, DAMPs not only produce weaker innate immune activation than counterpart PAMPs, but also induce less TLR signal desensitization, contribute to less innate immune cell death, and propagate more robust systemic coagulopathic effects than PAMPs. In this study, we elucidate in vitro and in vivo how DAMPs and PAMPs have a differential ability to activate innate immune signaling and the coagulation cascade and how local and systemic DAMPs and PAMPs distinctively cause organ injury. Finally, using distinctive immune stimulatory and procoagulative activities of DAMPs, we developed a potential early prognostic marker of late mortality and MOF in polytrauma patients.

\section{Results}

Necrotic cell- and bacteria-released molecules have differential innate immune stimulatory and procoagulative activities. To recapitulate cell death occurring after traumatic injury, we used sonication to induce ex vivo traumatic cell death because the sonication is known to cause acute necrosis followed by delayed apoptosis (19). Supernatants of sonicated fibroblasts contained various DAMPs, such as HMGB1 and extracellular DNAs (exDNAs) (Supplemental Figure 1; supplemental material available online with this article; https://doi.org/10.1172/ jci.insight.127925DS1), and various sizes of microparticles or extracellular vesicles (Supplemental Figure 2). These supernatants activated mouse macrophages and primary mouse cells (e.g., splenocytes and peripheral blood mononuclear cells) to produce proinflammatory cytokines such as TNF- $\alpha$, but negligible antiinflammatory cytokine IL-10 (Figure 1, A and B, and Supplemental Figure 3). Interestingly, supernatants of sonicated gram-negative bacteria induced multiple log-fold increase in production of both TNF- $\alpha$ and IL-10 by the macrophages compared with the supernatants of sonicated fibroblasts (Figure 1, C and D).

To prevent the unwanted onset of pathological inflammation, persistent stimulation of innate immune receptors is highly regulated by TLR tolerance mechanisms that lead to a hyporesponsiveness of TLRs to their cognate ligands as a result of a desensitization of TLR signaling by various self- and cross-regulatory mechanisms (20). Thus, innate immune cells pretreated with TLR stimulators could not fully respond to consecutive treatment with the same or other types of TLR stimulators. This is a potential mechanism by which immunoparalysis develops in sepsis patients and results in increased secondary infection and late mortality (21).

The supernatants of both sonicated fibroblasts and sonicated bacteria induced gradual suppression of inflammatory cytokine production and TLR downstream signaling molecule NF- $\mathrm{KB}$ activation in a dose-dependent manner after repeated exposure of innate immune cell and TLR reporter cells to the same supernatants (Figure 1, A-E). Sequential bacteria supernatant treatments completely abrogated the ability of innate immune cells to produce cytokines, whereas sequential fibroblast supernatant treatments partially suppressed innate immune cells to produce inflammatory cytokines. Consistent with sonicated bacteria and fibroblast supernatants, macrophages stimulated with TLR4-activating PAMP LPS were completely unresponsive to subsequent LPS treatment, whereas the macrophages stimulated with TLR4-activating DAMPs, such as heparan sulfate (HS) and HMGB1, were only partially tolerant to restimulation with HS and HMGB1 (Figure 1, F and G).

PAMP treatment, but not DAMP treatment induces innate immune cell death. TLR activation by PAMPs in an infected host is a major trigger of host immune responses against infection and clearance of pathogens, but also sequential TLR activation leads to dysfunction of the host immune system by multiple mechanisms, including TLR tolerance induction and innate immune cell cycle arrest and cell death (22, 23). However, it is still unclear whether DAMPs can induce innate immune cell cycle arrest and cell death as PAMPs do. Consistent with previous studies, the sonicated bacteria supernatants and LPS significantly induced mouse macrophage death and G0/G1 cell cycle arrest in a dose-dependent manner (Figure 2, A, C, E, and F).

Surprisingly, the sonicated fibroblast supernatants, HS, and HMGB1 did not significantly alter cell viability and cell cycle in mouse macrophages (Figure 2, B and D-F). These data suggest that TLR signaling tolerance, cell cycle arrest, and cell death may abrogate the ability of innate immune cells to induce innate immune and inflammatory responses upon sequential exposure to PAMPs. However, DAMP stimulation induces partial TLR signaling tolerance and minimal cell death and cell cycle arrest in innate immune cells, thereby leading to partial desensitization of innate immune cells to DAMP rechallenge. 
A

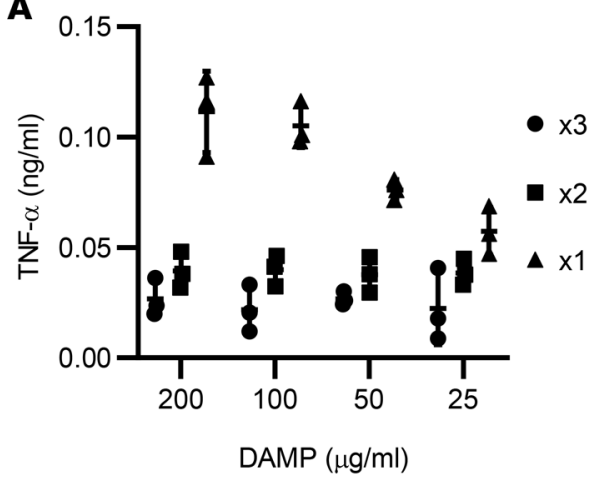

C

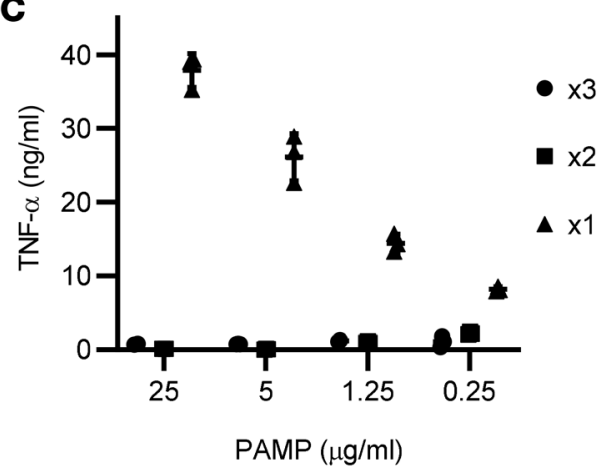

E

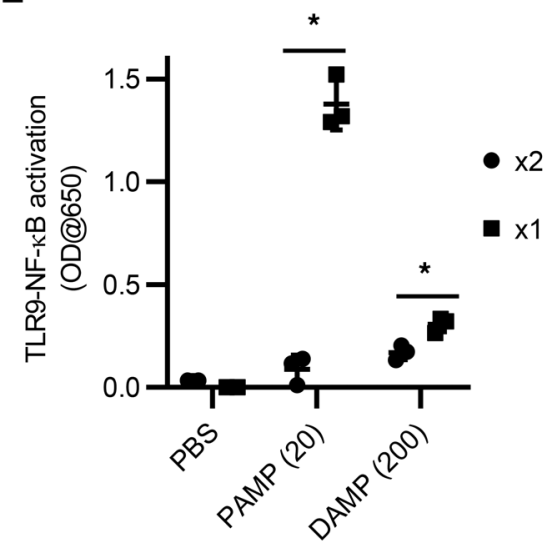

Stimulator $(\mu \mathrm{g} / \mathrm{ml})$
B

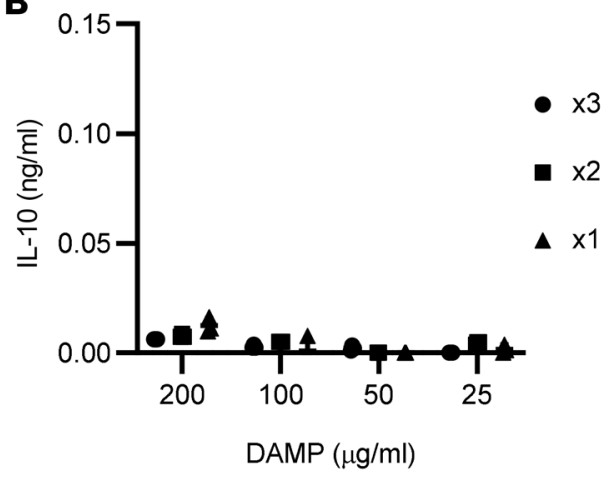

D

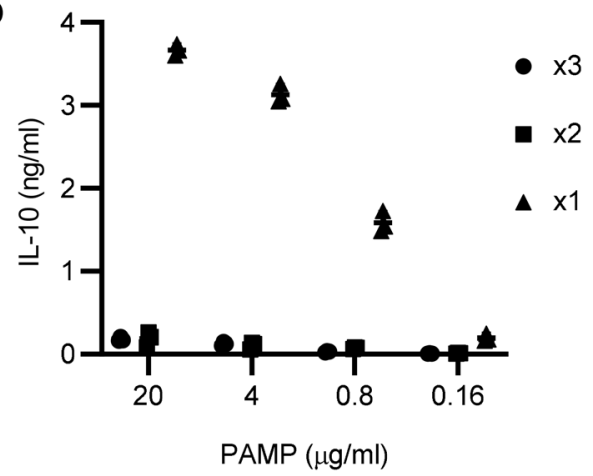

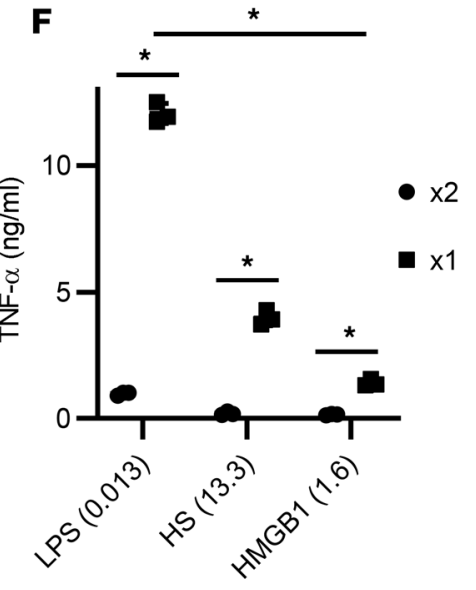

Stimulator $(\mu \mathrm{M})$
G
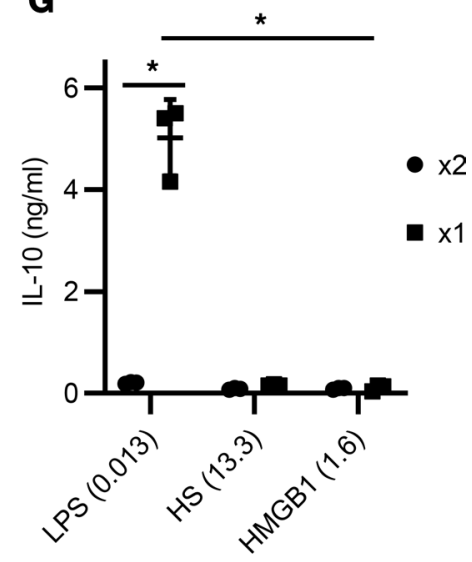

Stimulator $(\mu \mathrm{M})$

Figure 1. Comparison between the innate immune stimulation by DAMPs and PAMPs. Necrotic fibroblast supernatants (DAMPs) and necrotic gram-negative bacteria supernatants (PAMPs) generated by sonication were used as innate immune stimuli. (A-D) Macrophage cell line RAW264.7 was stimulated with (A and B) DAMPs or (C and $\mathbf{D})$ PAMPs at the indicated concentration. After first overnight incubation, macrophage culture supernatants were collected $(\times 1)$, followed by washing once and replenishing with fresh culture media. This procedure was repeated once $(\times 2)$ or twice $(\times 3)$. (E) TLR4-NF-KB-SEAP reporter cell line was stimulated with the DAMPs or PAMPs. The level of TLR4-mediated NF-KB activation was determined by measuring the amounts of secreted SEAP in reporter cell culture supernatants. (F and G) RAW264.7 cells were incubated with purified PAMPs (LPS) or DAMPs (HS, HMGB1), followed by washing and replenishing with fresh culture media. The amounts of TNF- $\alpha$ and IL-10 released from the cells after first $(\times 1)$ and second $(\times 2)$ incubation were measured. ${ }^{*} P<0.01$ (between indicated groups; Dunnett's multiple-comparisons test and paired $t$ test).

Local injection of PAMPs, but not DAMPs, cause systemic organ injury/dysfunction and mortality in mice. We next asked whether local and systemic injection of PAMPs and DAMPs lead to MOF and mortality in a murine model. Intraperitoneal and i.v. injection of LPS have been shown to induce systemic inflammatory responses, MOF, and mortality in murine models $(24,25)$. Consistent with LPS-induced MOF, intraperitoneal injection of sonicated bacteria supernatants elevated plasma TNF- $\alpha$ and IL- 6 levels in mice (Figure 3, A and B). These mice also gradually increased plasma alanine aminotransferase (ALT) and creatinine levels after injection, indicating liver and kidney damage (Figure 3, C and D). 


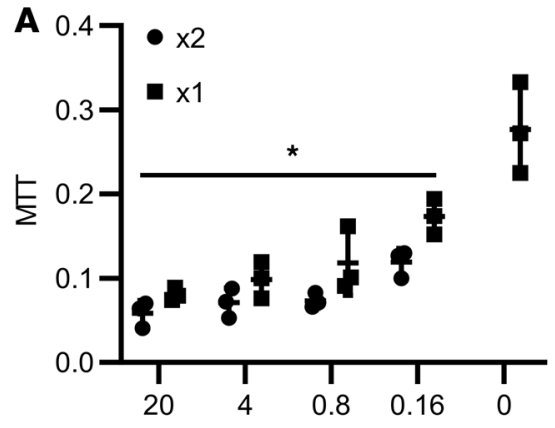

PAMP concentration $(\mu \mathrm{g} / \mathrm{ml})$

C
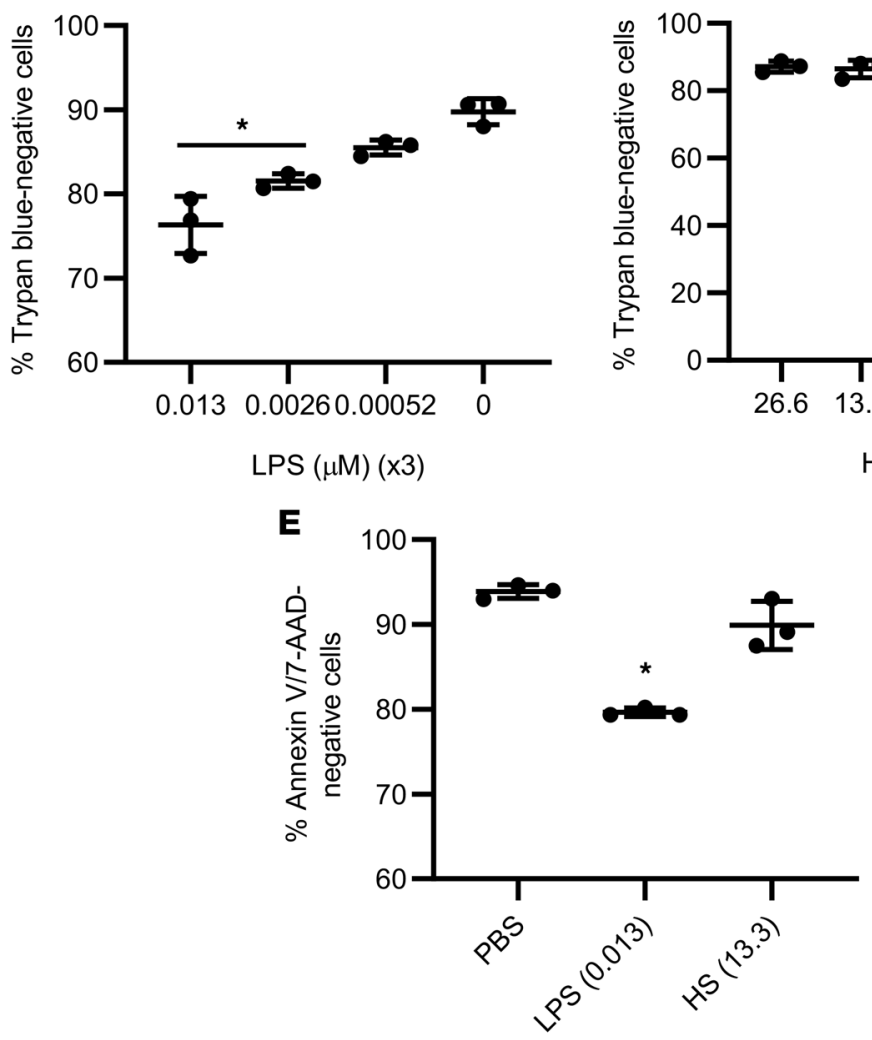

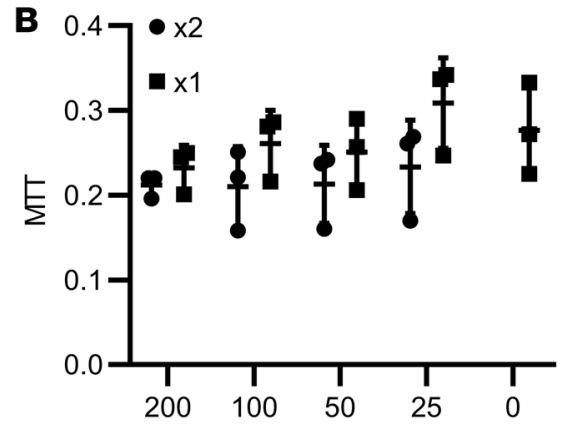

DAMP concentration $(\mu \mathrm{g} / \mathrm{ml})$

D

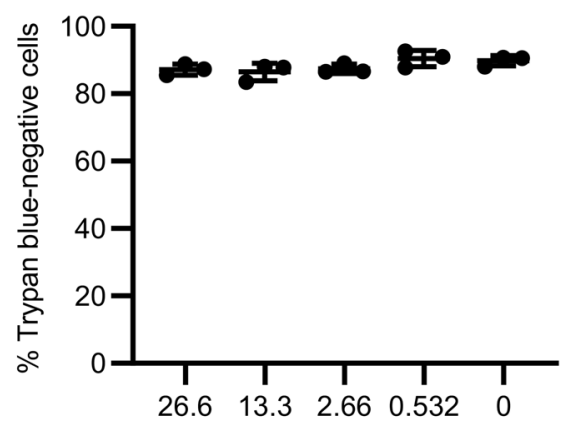

$\mathrm{HS}(\mu \mathrm{M})(\mathrm{x} 3)$

Figure 2. Macrophage cell death induction and growth inhibition by PAMPs, but not DAMPs. RAW264.7 cells were stimulated with PBS control, ( $\mathbf{A}$ and $\mathbf{F}$ ) necrotic gram-negative bacteria supernatants (PAMPs), (B and $\mathbf{F}$ ) necrotic fibroblast supernatants (DAMPs), (C, E, and F) LPS, or (D, E, and F) HS at indicated concentration. Fresh complete media supplemented with the stimuli were replenished every day for 1 to 2 days. After 3 days after first stimulation, (A and B) cell growth inhibition (MTT) and cell death (C and $\mathbf{D})$ trypan blue staining and (E) annexin V/7-AAD staining were determined. (F) After first stimulation with the stimuli, cells were stained with propidium iodide, followed by cell cycle analysis using flow cytometry. ${ }^{*} P<0.05$ (vs. untreated or PBS; Dunnett's multiple-comparisons test).

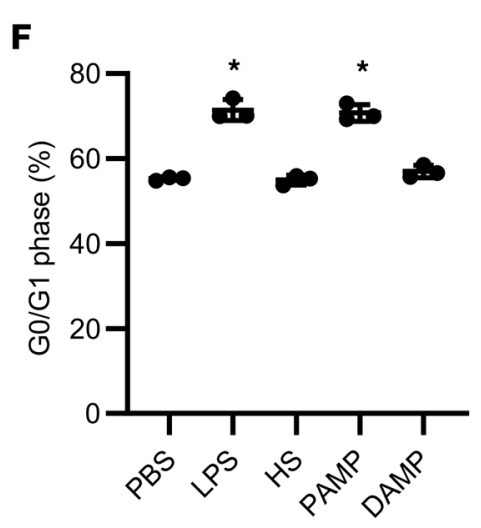

Stimulator $(\mu \mathrm{M})(\mathrm{x} 3)$
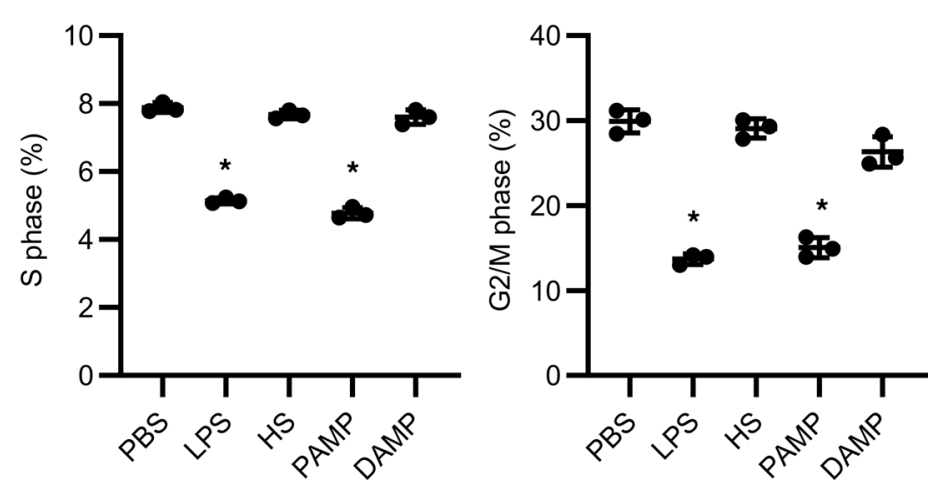

In addition, plasma exDNA and thrombin-antithrombin complex (TATc) levels gradually rose in the mice with intraperitoneal injection of bacteria supernatants (Figure 3, E and F).

By contrast, intraperitoneal injection of sonicated fibroblast supernatants did not significantly raise the levels of TNF- $\alpha$, IL-6, ALT, and creatinine (Figure 3). Mice treated with these supernatants demonstrated 

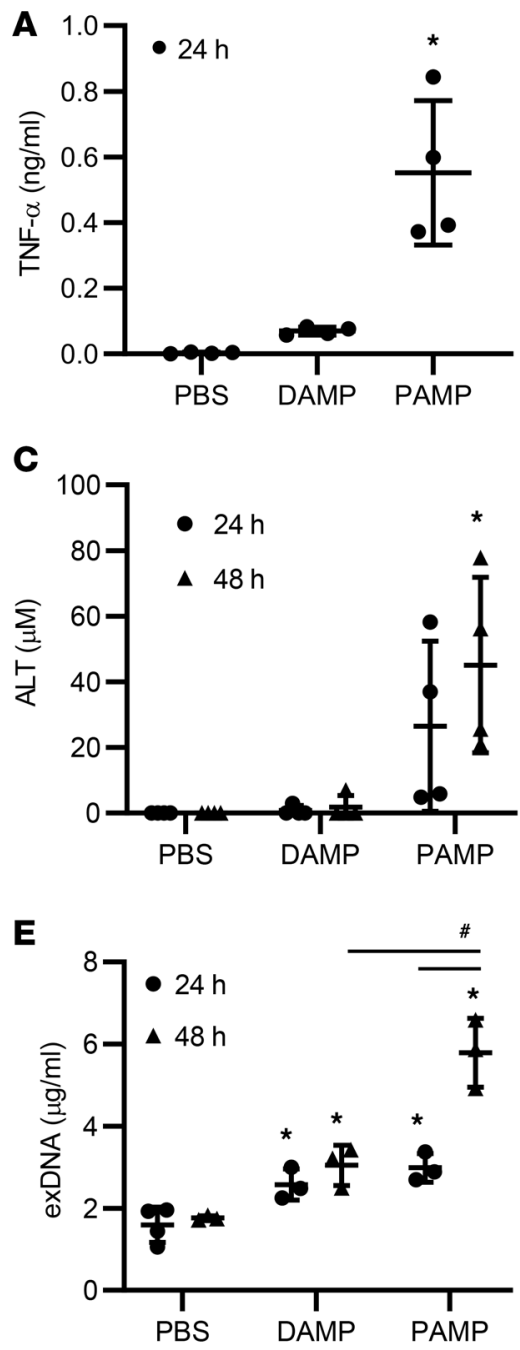
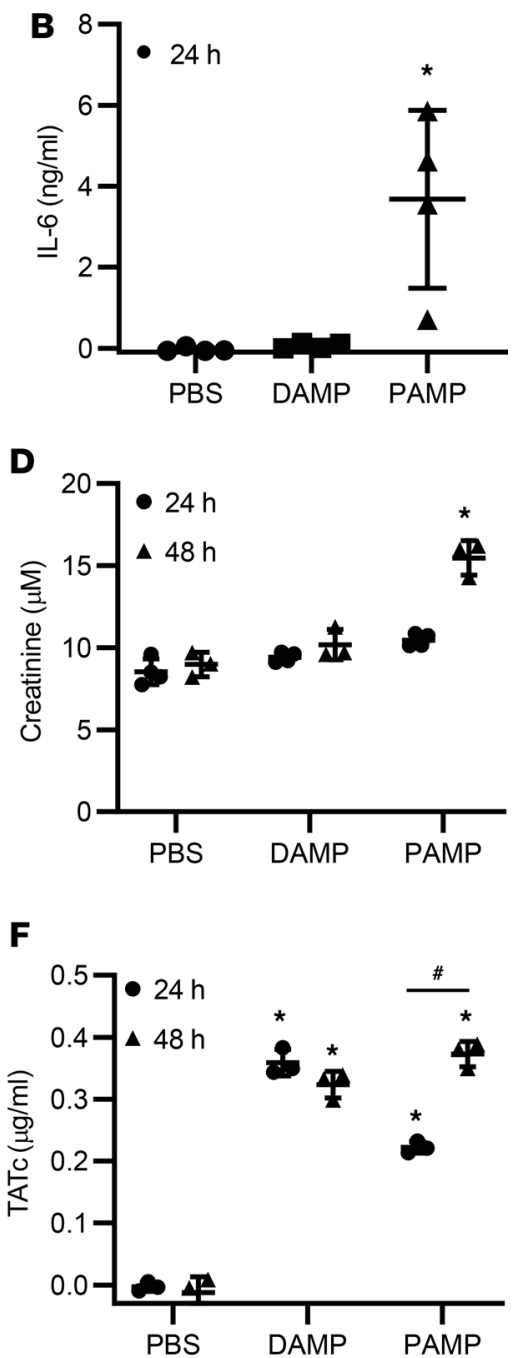

Figure 3. Local injection of PAMPs, but not DAMPs, induces systemic inflammation and organ damage in mice. Mice were injected intraperitoneally with PBS control, necrotic fibroblast supernatants (DAMPs) $(1500 \mu \mathrm{g})$, or necrotic gram-negative bacteria supernatants (PAMPs) $(1500 \mu \mathrm{g})$. (A-F) Plasma was collected at 24 and/or 48 hours after injection. Plasma markers of ( $\mathbf{A}$ and B) systemic inflammation (TNF- $\alpha$ and IL-6), (C) liver injury (ALT), (D) kidney dysfunction (creatinine), (E) DAMP generation (exDNA), and (F) thrombosis (TATc) were determined. (G) Mortality was monitored every day. $n=5 .{ }^{*} P<0.05$ (vs. PBS). ${ }^{\#} P$ $<0.05$ (between indicated groups; Kruskal-Wallis test and log-rank test).

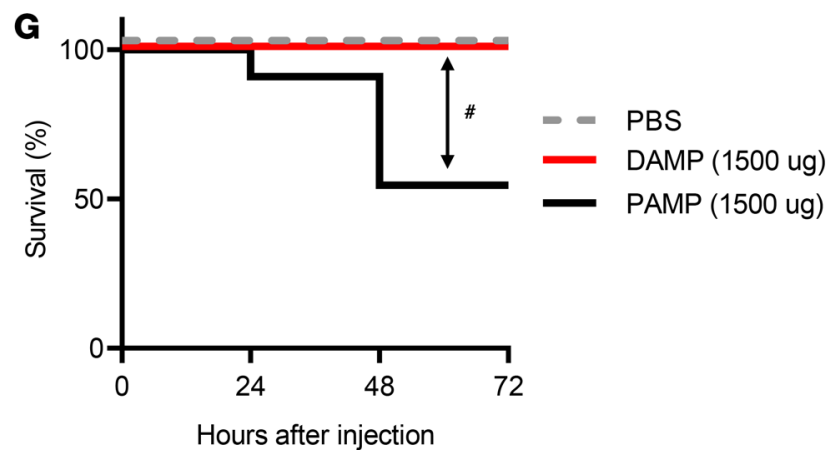

increased exDNA and TAT levels in the bloodstream 24 hours after treatment, although the levels of exDNA were significantly lower than in mice treated with sonicated bacteria (Figure 3E). In addition, mice treated with sonicated fibroblast supernatant did not show an increase in levels of exDNA and TATc from 24 hours to 48 hours, whereas mice treated with sonicated bacteria demonstrated a significant increase over this interval (Figure 3, E and F). Intraperitoneal injection of sonicated bacteria supernatants killed $50 \%$ of mice within 48 hours, but intraperitoneal injection of sonicated fibroblast supernatants did not cause any morbidity and mortality in mice (Figure 3G).

Systemic injection of DAMPs leads to greater mortality compared with PAMPs. Because circulating DAMP levels are often correlated with trauma mortality and MOF incidence (10), we asked whether systemically injected DAMPs differentially cause mortality and MOF in mice compared with locally injected DAMPs. 
A
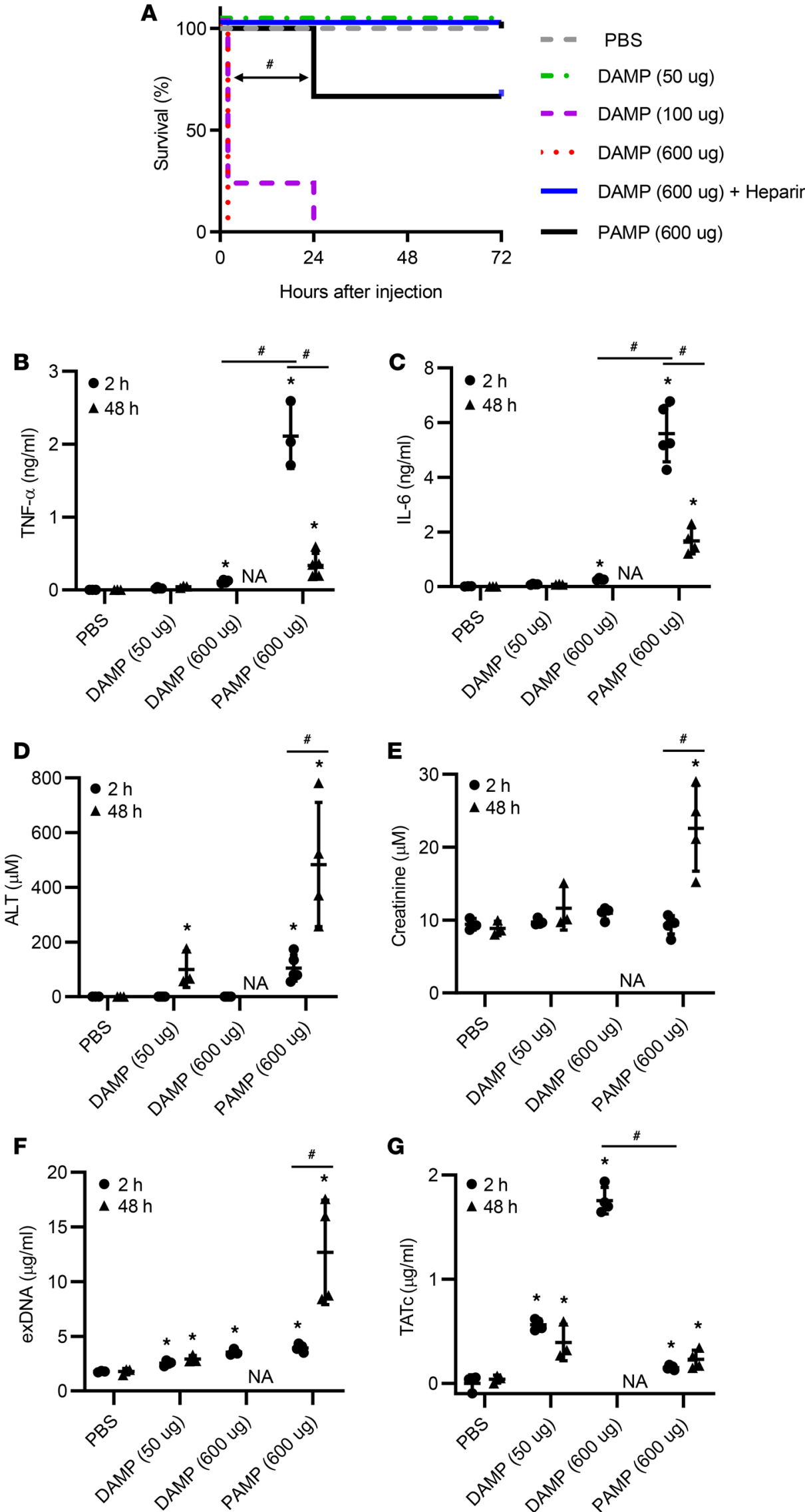
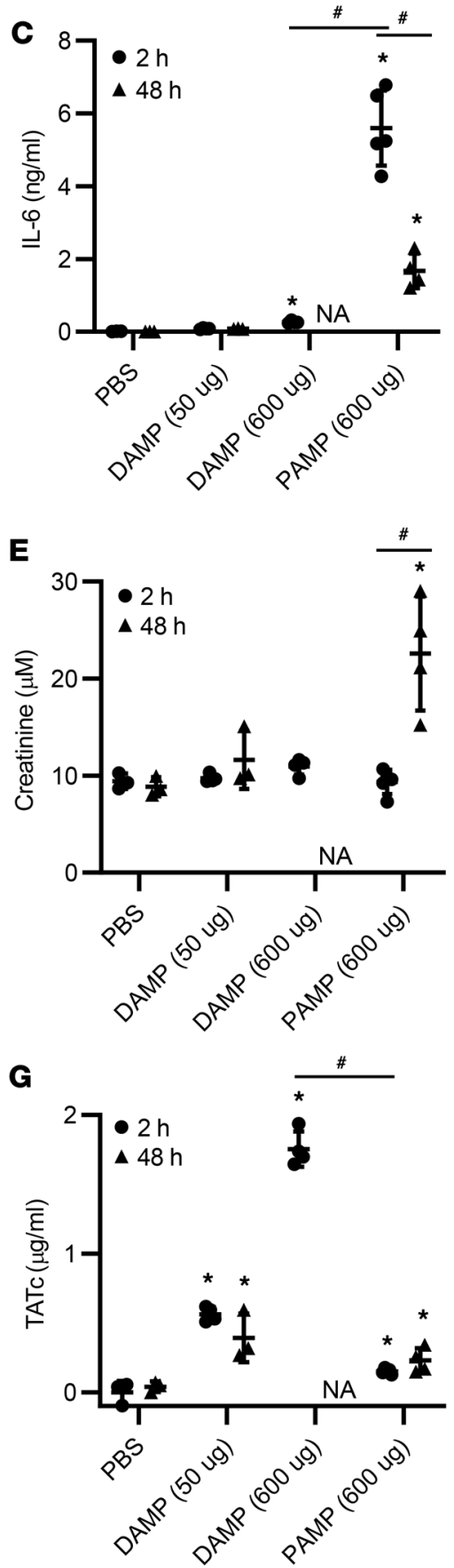

Figure 4. Single systemic injection of PAMPs and DAMPs differentially induces mortality and organ damage in mice. Mice were i.v. injected with PBS control $(n=4)$, necrotic gram-negative bacteria supernatants (PAMPs) $(n=6,600 \mu \mathrm{g})$, or necrotic fibroblast supernatants (DAMPs) $(n=5,600$ $\mu \mathrm{g})$ in the presence or absence of heparin. (A) Mortality was monitored every day. (B-G) Plasma was collected at 2 and 48 hours after injection. Plasma levels of (B) TNF- $\alpha$, (C) IL-6, (D) ALT, (E) creatinine, (F) exDNA, and (C) TATc were determined. ${ }^{*} P<0.05$ (vs. PBS). $\# P<0.05$ (between indicated groups; Kruskal-Wallis test and log-rank test). NA, not applicable.

Intravenous injection of sonicated bacteria supernatants led to a similar mortality rate as intraperitoneal injection (Figure 4A). Interestingly, unlike when administered by intraperitoneal injection, i.v. injection of sonicated fibroblast supernatants acutely killed mice in a dose-dependent manner mostly by inferior vena cava (IVC) thrombosis, and this acute mortality was prevented by treatment with anticoagulant heparin (Figure 4A). Surprisingly, sonicated fibroblast supernatant injection caused worse increased mortality compared with sonicated bacteria supernatant injection.

Consistent with intraperitoneal administration of sonicated bacteria and fibroblast supernatants, i.v. injection of sonicated bacteria supernatants immediately produced greater levels of TNF- $\alpha$ and IL- 6 in the blood of mice than i.v. injection of sonicated fibroblast supernatants (Figure 4, B and C). These TNF- $\alpha$ and IL-6 levels significantly decreased at 48 hours after injection. In contrast, plasma ALT and creatinine levels significantly increased from 2 hours to 48 hours after injection of sonicated bacteria supernatants (Figure 4, D and E). Sonicated fibroblast supernatants also led to an increase in the plasma ALT level, but not creatinine level, at 48 hours after injection compared with PBS. Moreover, i.v. injection of sonicated bacteria supernatants led to an increase in exDNA and TATc levels in the blood over time, similar to ALT and creatinine elevation (Figure 4, F and G). Interestingly, i.v. injection of sonicated 
A

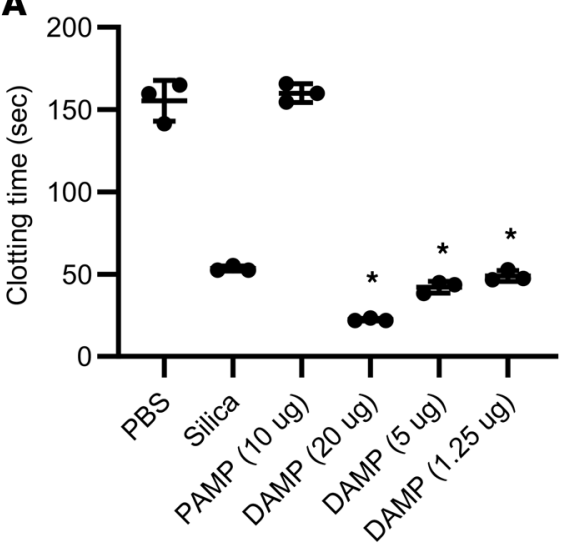

C
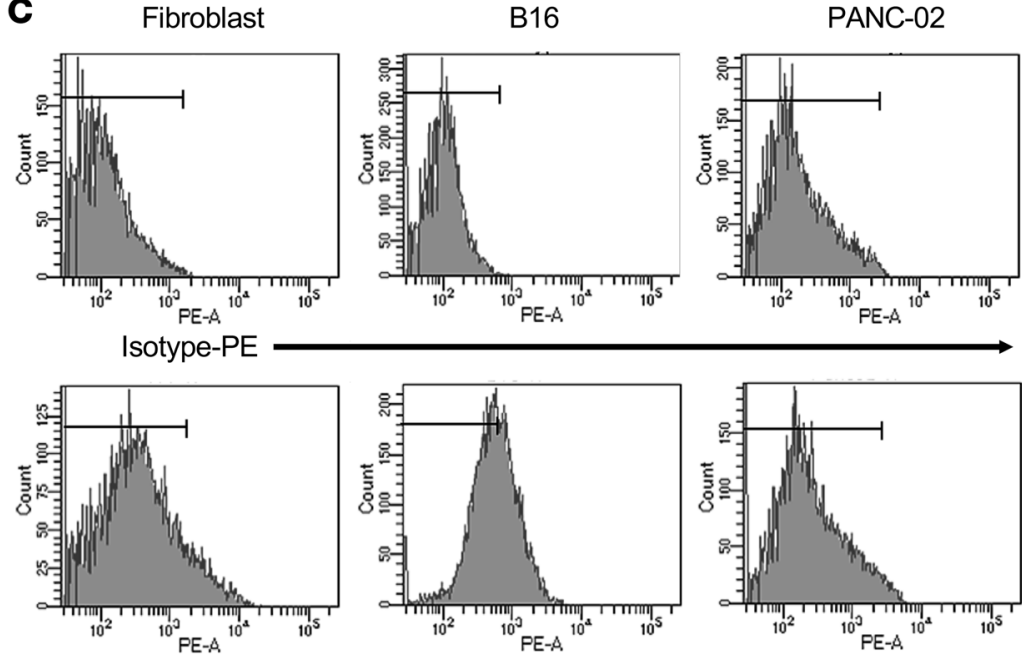

Anti-CD142-PE

D

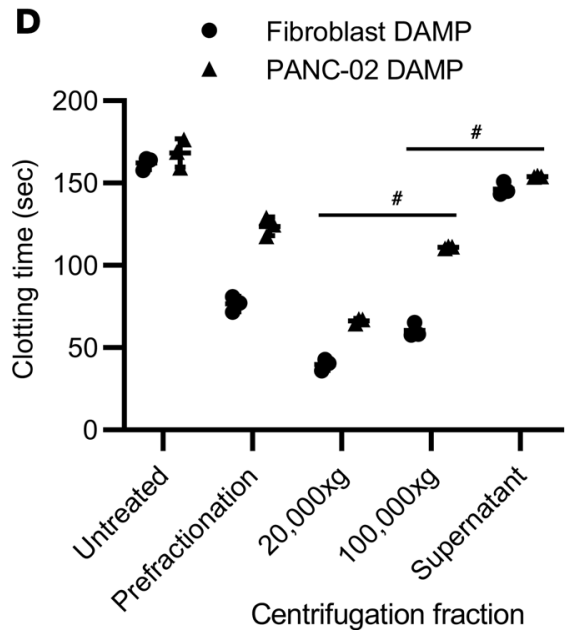

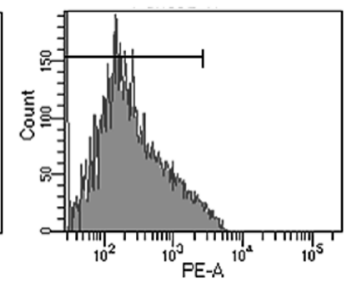

B

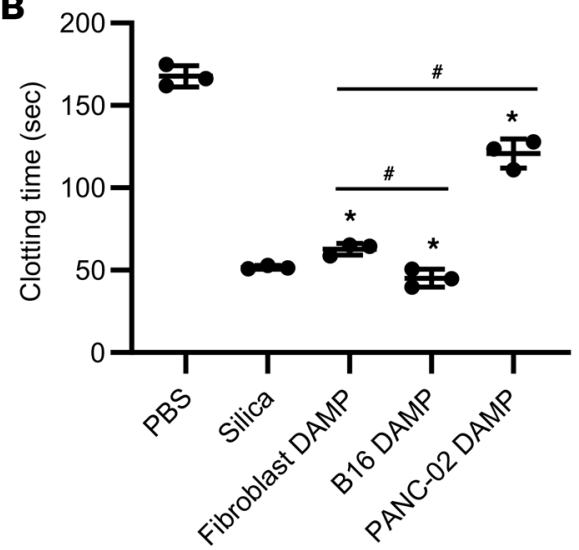

ANC-02

\section{E}
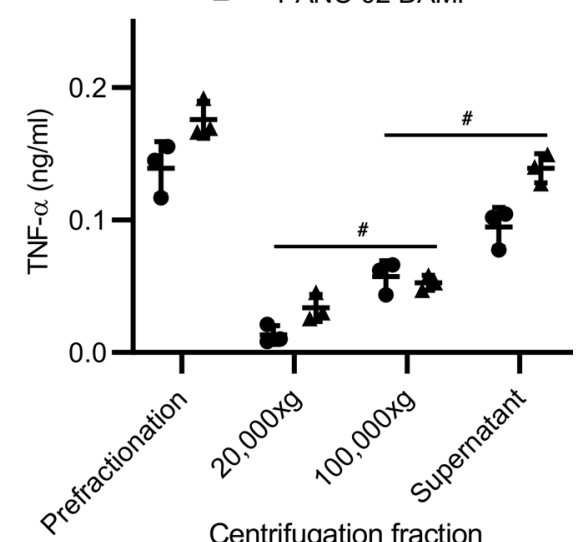

Centrifugation fraction
Figure 5. Necrotic cell supernatants, but not necrotic bacteria supernatants contain procoagulants. Supernatants of sonicated normal fibroblasts, B16 melanoma cells, and PANC-02 pancreatic cancer cells were used as DAMPs. Supernatants of sonicated Pseudomonas aeruginosa were used as PAMPs. (A and B) Normal mouse plasma was stimulated with PAMPs or DAMP, followed by measuring clotting time. PBS and silica were used as negative and positive controls. (A) Clotting time of normal mouse plasma (50 $\mu \mathrm{L})$ stimulated with DAMPs $(1.25,5$, or $20 \mu \mathrm{g})$ from fibroblasts or PAMPs $(10 \mu \mathrm{g})$ was measured using a coagulometer. (B) Normal mouse plasma $(50 \mu \mathrm{L})$ was stimulated with DAMPs $(5 \mu \mathrm{g})$ from fibroblasts, B16 cells, and PANC-02 cells. (C) TF (CD142) expression on the fibroblasts, B16 cells, and PANC-02 cells was determined using flow cytometry. (D and E) Large particle (3,000-20,000-g fraction), small particle $(20,000-100,000-g$ fraction), and supernatant fractions were isolated from necrotic cell supernatants by differential centrifugations. (D) Normal mouse plasma $(50 \mu \mathrm{L})$ was stimulated with unfractionated cell supernatants, large particle, small particle, and supernatant fraction $(5 \mu \mathrm{g})$, followed by measuring clotting time. (E) RAW264.7 cells were stimulated overnight with the fractions $(100 \mu \mathrm{g} / \mathrm{mL})$. ${ }^{*} P<0.05$ (vs. PBS; Dunnett's multiple-comparisons test). ${ }^{*} P<0.05$ (between indicated groups; Tukey's multiple-comparisons test).

fibroblast supernatants produced a logfold higher TATc level at 2 hours compared with i.v. injection of sonicated bacteria supernatants (Figure 4G).

$D A M P$ s, but not PAMPs, activate blood coagulation cascade in a tissue factor- and size-dependent manner. Unlike sonicated bacteria supernatants that caused mortality and MOF in mice after intraperitoneal or i.v. injection, sonicated fibroblast supernatants caused mortality and MOF in mice only if they were i.v. administered. Interestingly, mice with sonicated fibroblast supernatants had higher levels of plasma TATc and lower levels of plasma inflammatory cytokines than mice with sonicated bacteria supernatants (Fig-

ure 4). Moreover, the sonicated fibroblast supernatant-caused mortality in mice was completely prevented by pretreatment with heparin (Figure 4A). Thus, we postulated that sonicated fibroblast supernatants, but not sonicated bacteria supernatants, would contain potent procoagulants.

To answer this question, we compared activated plasma coagulation time between mouse plasma treated with sonicated fibroblast supernatants and mouse plasma treated with sonicated bacteria supernatants. The sonicated fibroblast supernatant treatment accelerated plasma clotting time in a dose-dependent manner, but the sonicated bacteria supernatants did not (Figure 5A). Transmembrane protein tissue factor (TF) is constitutively expressed on the surface of subendothelial cells, such as fibroblasts, 
Table 1. Trauma patient characteristics

\begin{tabular}{|c|c|c|c|c|c|c|}
\hline Patient & Age & Sex & Injury & MOF & ISS & Mortality \\
\hline 2 & 25 & $M$ & MVC & $\mathrm{N}$ & 27 & $\mathrm{~N}$ \\
\hline 4 & 18 & $\mathrm{~F}$ & Fall $<15 \mathrm{ft}$ & $\mathrm{N}$ & 20 & $\mathrm{~N}$ \\
\hline 5 & 22 & M & GSW & Y & 41 & $\mathrm{~N}$ \\
\hline 7 & 68 & M & MVC & Y & 50 & Y (day 5) \\
\hline 13 & 70 & M & Fall $<15 \mathrm{ft}$ & $\mathrm{N}$ & 18 & $\mathrm{~N}$ \\
\hline 16 & 62 & M & MVC & $\mathrm{Y}$ & 41 & $\mathrm{~N}$ \\
\hline 17 & 22 & M & MVC & $\mathrm{N}$ & 9 & $\mathrm{~N}$ \\
\hline 23 & 54 & M & MCC & $\mathrm{N}$ & 29 & $\mathrm{~N}$ \\
\hline 44 & 77 & $M$ & MVC & $\mathrm{Y}$ & 50 & $\mathrm{~N}$ \\
\hline
\end{tabular}

CSW, gunshot wound; ISS, injury severity score; MOF, multiple organ failure; MVC, motor vehicle collision.

smooth muscle cells, and pericytes, which are not exposed to blood until tissue or vessel damage occurs (26). Upon tissue injury, TF triggers the clotting process. Thus, we determined whether TF expression level was correlated with the coagulability of necrotic cells. To answer this question, 3 different cells that express high, low, and negligible levels of TF were sonicated, followed by isolation of supernatants. The procoagulant activity of these supernatants was positively correlated to the amount of TF surface expression on the cells (Figure 5, B and C).

Interestingly, supernatants isolated from sonicated PANC-02 cells that express no or marginal levels of TF significantly activated mouse plasma coagulation compared with PBS control (Figure 5B), which suggests that TF may not be the only procoagulant present in the necrotic cells. Extracellular nucleic acids have been shown to induce thrombosis in vitro and in vivo (27). exDNA levels are high in the sonicated cell supernatants (Supplemental Figure 1B). Thus, we determined whether DNAs and RNAs in the sonicated cell supernatants would activate plasma coagulation. To answer this question, sonicated fibroblast and PANC-02 cell supernatants were pretreated with nucleases, followed by activation of plasma coagulation. DNase and RNase pretreatments did not significantly alter the coagulation activity of sonicated cell supernatants. Moreover, naked long double-stranded RNAs and genomic DNAs did not activate plasma coagulation (Supplemental Figure 4). Interestingly, large particle fraction of sonicated cell supernatants had greater procoagulative activities and less innate immune stimulatory activities than small particle and non-particle fractions of sonicated cell supernatants (Figure 5, D and $\mathrm{E}$ ). These data suggest that to activate coagulation cascade, procoagulants released from necrotic cells need to be present on a membranous surface.

Persistent circulation of DAMPs in the bloodstream induce systemic inflammation and organ injury/dysfunction. A growing body of evidence shows that circulating DAMP levels are correlated to the SIRS and MOF in patients with trauma (28). In addition, persistent organ dysfunction is predictive of morbidity and late mortality after severe trauma (29). Given that TLR-activating DAMPs cause systemic inflammatory response and MOF, we hypothesized that persistent circulation of TLR-activating DAMPs is required to develop persistent organ damage/dysfunction, thereby causing worse morbidity and late mortality. To answer this question, we performed i.v. daily injections of sonicated cell supernatants into mice and determined whether persistent administration of such cell death byproducts caused systemic inflammatory responses and MOF.

Because sonicated fibroblasts released very potent procoagulants that caused acute toxicity, we used sonicated PANC-02 cell supernatants that have mild procoagulative activity and comparable innate immune stimulatory activity to sonicated fibroblast supernatants (Figure 5, D and E). Intravenous injection of high-dose sonicated PANC-02 cell supernatants killed mice immediately after injection, mostly but not exclusively, by IVC thrombosis; however, i.v. injections of low-dose sonicat- 
A

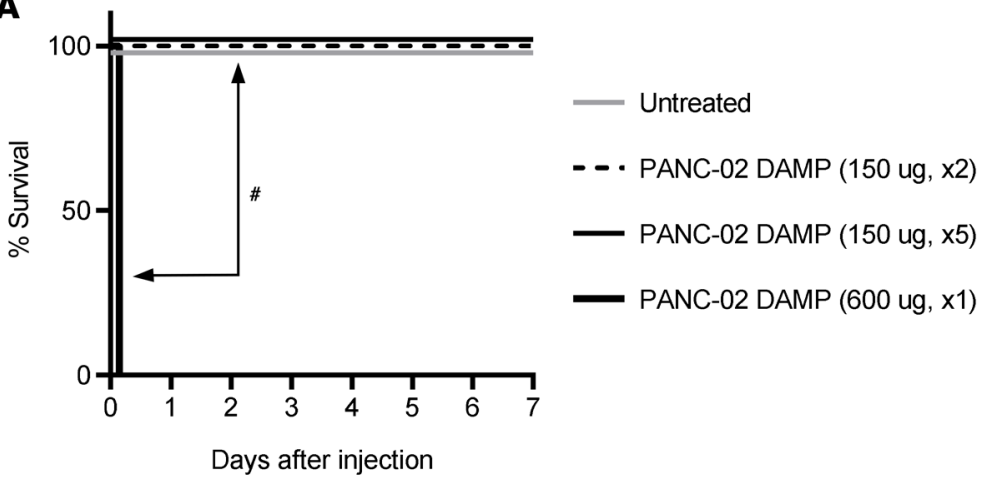

C

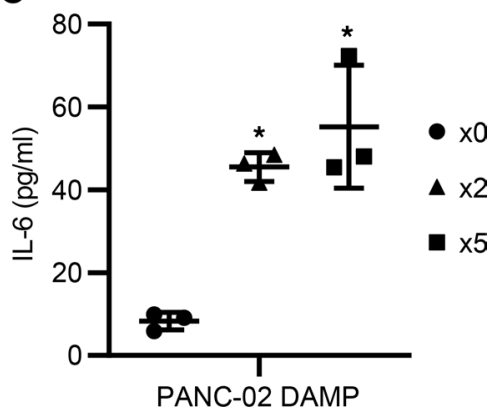

E

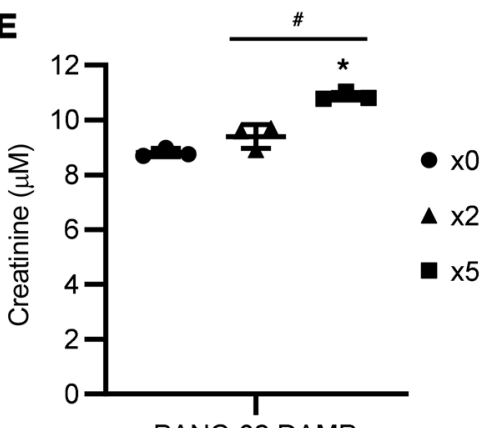

PANC-02 DAMP

G

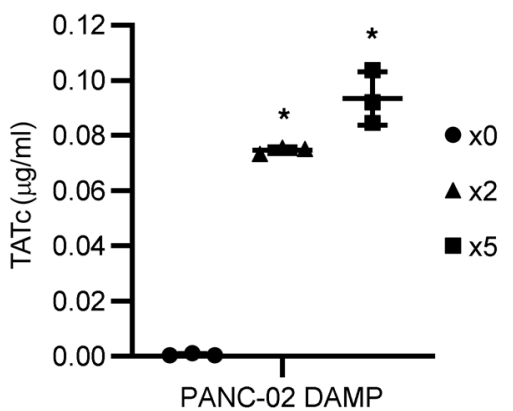

Figure 6. Multiple systemic injections of low procoagulative DAMPs gradually increase proinflammatory cytokines and organ damage markers in mice. PANC- 02 cells were sonicated, followed by harvesting DAMP-containing supernatants. Mice $(n=5)$ were i.v. injected every day for 1-5 days with DAMPs $(150 \mu \mathrm{g}$ or $600 \mu \mathrm{g})$. (A) Mortality was monitored every day. (B-G) Plasma was collected on days 0 , 2, and 5. Plasma levels of (B) TNF- $\alpha$, (C) IL-6, (D) ALT, (E) creatinine, (F) exDNA, and (G) TATc were determined. ${ }^{*} P<$ 0.05 (vs. untreated). ${ }^{\#} P<0.05$ (between indicated groups; Kruskal-Wallis test and log-rank test).

ed PANC-02 cell supernatants did not cause early and late mortality in these mice (Figure 6A). Daily systemic injections of low-dose sonicated PANC02 cell supernatants gradually elevated the levels of TNF- $\alpha$, IL- 6, ALT, creatinine, exDNAs, and TATc in the blood of mice in a duration-dependent manner (Figure 6, B-G).

Circulation of procoagulative particles early after injury is a potential early prognostic marker of MOF and late-onset mortality in trauma patients. Given that proinflammatory and prothrombotic DAMPs released from the initial insult can cause secondary tissue damage, leading to de novo DAMP release, the levels of proinflammatory and procoagulative DAMPs in the blood of trauma patients early after injury could be early predictive markers of MOF and late-onset mortality in these patients. To address this question, we determined the correlation between the levels of circulating immunostimulatory DAMPs and procoagulative particles and the MOF incidence and mortality in polytrauma patients (Table 1). Because most endogenous DAMPs are unknown, the levels of total innate immune stimulators in the patient's plasma were indirectly determined using TLR reporter cells, as described previously (10).

Plasma isolated from the blood of trauma patients induced elevated NF- $\kappa B$ activation in TLR reporter cells compared with plasma isolated from the blood of normal healthy controls. Interestingly, the levels of plasma-activated TLR-NF- $\mathrm{KB}$ tended to gradually rise after injury, mostly but not exclusively, in trauma patients with MOF and/or late-onset mortality (Supplemental Figure 5). The levels of plasma-activated TLR-NF- $\mathrm{BB}$ at late, but not early time points after injury were significantly different between trauma patients with MOF and healthy people (Figure 7, A-D). How-

ever, the levels of plasma-activated TLR-NF- $\mathrm{B}$ were not significantly different between trauma patients with or without MOF and late mortality at any time points after trauma. Moreover, the amounts of total particles circulating in the blood of trauma patients on day 1 after injury were not significantly different between the trauma patients with or without MOF (Figure 7E). Surprisingly, procoagulative activity of circulating particles in trauma patients with MOF and late death was significantly higher than that of particles in trauma patients without MOF and late death and healthy persons (Figure 7F). 
A

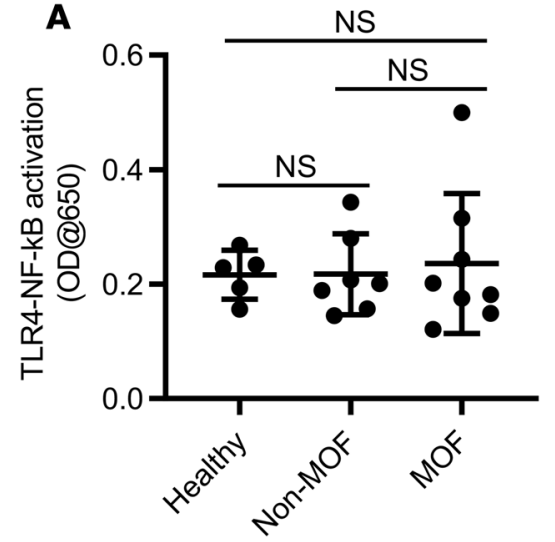

C
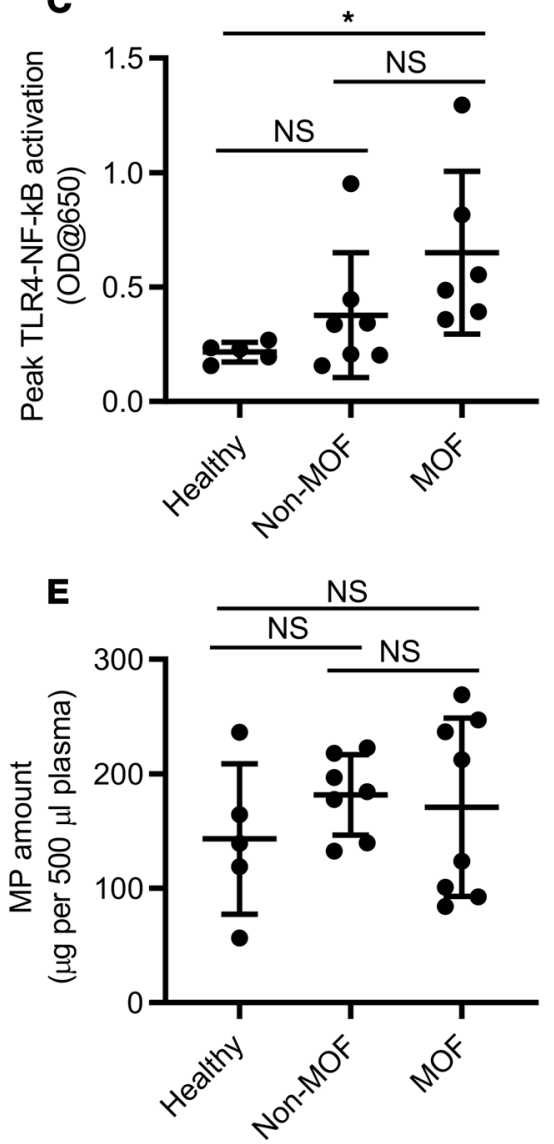
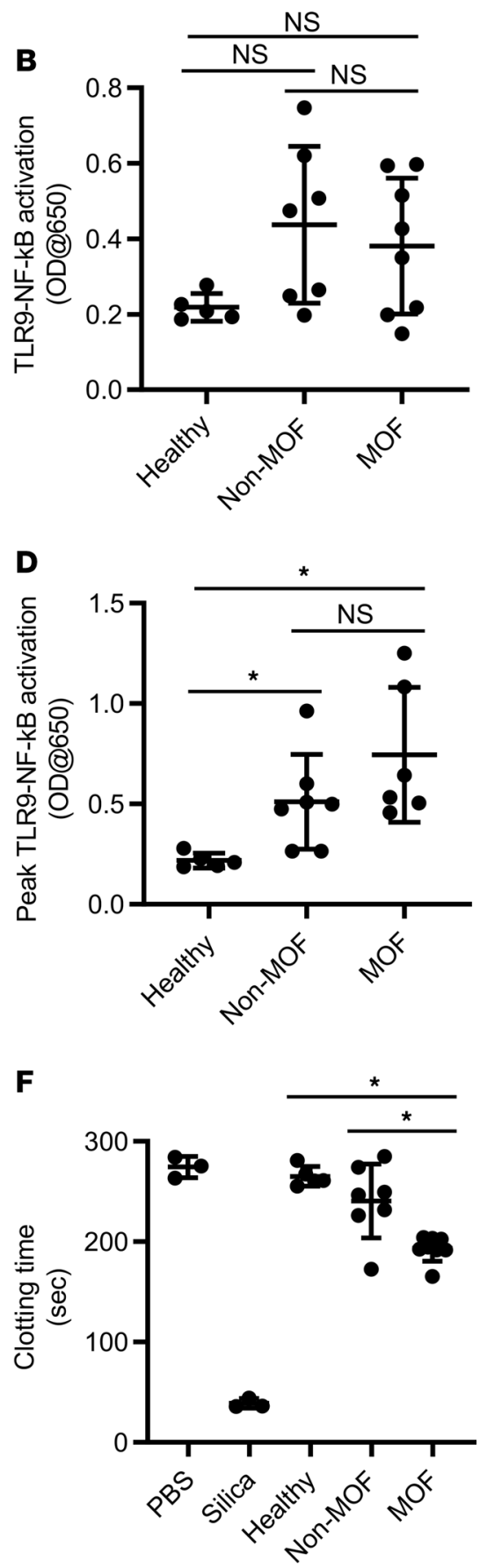

Figure 7. Early presence of circulating procoagulative particles and TLR-activating DAMPs after sterile injury predicts late-onset multiple organ failure (MOF) and mortality in trauma patients. (A-D) HEK-TLR4 and HEK-TLR9 reporter cells were stimulated overnight with serum $(20 \%$ $v / v)$ isolated from healthy persons $(n=5)$ or polytrauma patients without MOF and mortality $(n=7)$ or with MOF $(n=8)$ at multiple time points after injury. (A and $\mathbf{B}$ ) Serum isolated day 1 after trauma. (C and $\mathbf{D})$ Sera were isolated at peak TLR-activating time point after trauma. (E and F) 3,000-100,000-g particle fraction was purified from $500 \mu \mathrm{L}$ plasma isolated from healthy persons $(n=5)$ or trauma patients at 1 day after injury. Trauma patients without MOF and mortality $(n=7)$ and trauma patients with MOF $(n=8)$ were included in this study. (E) Total amounts of 3,000-100,000- $g$ particle fraction was measured by BCA protein assay. (F) Normal human plasma $(50 \mu \mathrm{L})$ was stimulated with the particle fraction $(15 \mu \mathrm{g})$, following by measuring plasma clotting time. ${ }^{*} P<0.05$ (between indicated groups; Kruskal-Wallis test). NS, not significant.

\section{Discussion}

Innate immune responses and coagulation are a frontline mechanism by which the body heals damaged tissues and protects a wounded area from infection. However, when left unchecked, the innate immune system and coagulation cascade interact synergistically to cause morbidity and mortality after trauma and sepsis $(30,31)$. In this study, we demonstrate that DAMPs contribute differently than PAMPs to the pathogenesis of MOF and mortality because of several factors.

We first showed that unpurified and purified DAMPs and PAMPs generated from damaged cells and bacteria induce a differential inflammatory response when innate immune cells are stimulated in vitro. Compared with PAMPs, DAMPs only induced a marginal inflammatory cytokine TNF- $\alpha$ production and did not induce any antiinflammatory cytokine IL-10 production. Moreover, DAMPs are less capable of desensitizing innate immune receptors to a repeated stimulus. At the highest concentration of PAMPs, we observed near- 
ly complete paralysis of both inflammatory and antiinflammatory cytokine production after repeated exposure, whereas innate immune cells exposed to repeated DAMP challenges demonstrated a reduced ability to respond but did not fully desensitize to the DAMPs. This would support a previously studied host protective mechanism known as endotoxin tolerance in which innate immune cells enter into a transiently dormant state to protect against pathologic derangement of inflammation upon repeated exposure to endotoxin (21).

Other studies have demonstrated that the TLR4-activating DAMP HMGB1 desensitized TLR4 signaling upon repeated HMGB1 challenge (32). Consistent with previous studies, we observed that HMGB1, HS, and unpurified cell death byproducts significantly induced TLR signaling tolerance. However, these HMGB1, HS, and unpurified cell death byproducts induced incomplete TLR signaling tolerance whereas LPS and bacteria death byproducts induced complete TLR signaling tolerance. This discrepancy is mostly, but not exclusively, the result of the difference in TLR signal strength and cell death induction between DAMPs and PAMPs. This study suggests that DAMPs may differentially contribute to the desensitization of innate immune cells to persistent insults compared with PAMPs.

Prolonged inflammatory and immune suppressive responses and nosocomial infections are well-known contributors to morbidity and mortality in ICUs (33). Our data suggest that DAMPs and PAMPs may differentially contribute to induction of inflammatory complications and secondary infection after trauma. In vivo animal data further supported our initial findings of PAMPs' ability to induce a more potent inflammatory response. Not surprisingly, even after intraperitoneal injection of PAMPs, a clear systemic inflammatory response was elicited and led to gradual organ dysfunction and ultimately death of $50 \%$ of the mice in this cohort. This finding closely resembles the long-held belief in a transition from a local inflammatory response to systemic derangement and organ failure in the setting of sepsis (34). Even though systemic inflammatory responses did not develop in mice with a single DAMP injection, the fact that TATc levels were also elevated in the plasma of both DAMP- and PAMP-treated mice at 48 hours demonstrates a link between the innate immune response and coagulation cascade.

This link between innate immune response and coagulation is a relatively new perspective on host defense mechanism against infection and injury. When exposed to DAMPs or PAMPs, innate immune cells located in the intravascular space retain the ability to deliver activated intravascular TF, activating the extrinsic coagulation pathway. Historically, this has been viewed as a host protective mechanism against invading pathogens but can also cause pathologic thrombosis and further tissue injury via ischemia when left unchecked (35). The contribution of DAMPs to tissue micro-ischemic injury is likely underappreciated; as demonstrated in a rat model, HMGB1 exposure was associated with increased glomerular deposition of fibrin and worse mortality outcomes (36). Although it is known that activated innate immune cells have the propensity to cause pathologic coagulation (37), our study would suggest that DAMP-mediated micro-ischemic injury may contribute to de novo DAMP synthesis propagating a vicious cycle of tissue damage resulting in $\mathrm{MOF}$.

These in vivo findings were further supported with a dramatic difference after systemic injection of DAMPs and PAMPs. Bacterial byproducts caused a robust early inflammatory response as would be expected in bacteremia and subsequent cytokine storm. Again, gradual organ dysfunction over the first 2 days after bacterial insult was observed similar to MOF progression in sepsis (34). However, what is somewhat unexpected is the rapid rise in TATc observed after i.v. injection of cell death byproducts. All animals injected with the sonicated fibroblast supernatants at high dose died as a result of massive central venous thrombus shortly after administration. This stark and immediate difference in mortality in the systemically injected DAMP group of mice suggests an incomplete understanding of DAMP pathophysiology. Because we accounted for the traditional TF pathway and extracellular nucleic acids known clotting effects, it is likely that a subset of molecules released from physiologic normal cells under stress or after traumatic injury, in combination with microparticles in the intravascular space, activate potently with the coagulation cascade (38). These molecules have the potential to be early therapeutic targets for the morbidity and mortality of trauma patients.

This is an important finding because it may lead to further prognostication of patients sustaining severe polytraumatic injury who survive early resuscitative measures and are susceptible to late complications such at MOF or nosocomial infection. As was shown when we examined plasma samples of trauma patients, TLR-activating molecules are more prevalent compared with healthy controls. Our study suggests that plasma TLR stimulators may not be the only important factor in the systemic physiologic derangement contributing to the morbidity and mortality of trauma patients. The procoagulative activity of plasma DAMPs may be equally contributory. Using distinctive immune stimulatory and procoagulative activities of DAMPs, we developed a potential early prognostic marker of late mortality and MOF in traumatically injured patients. 
This study has the potential to broadly impact the field of trauma and resuscitation medicine. It is important to note that the activity levels, both procoagulative and immune stimulatory, of DAMPs in circulation may be more important than the sheer volume of molecules because some will be physiologically inert. Future studies will work to expand upon the patient population, incorporating critically ill patients from a variety of different mechanisms. Specifically, targeting procoagulative microparticles and elucidating mechanisms by which trauma releases active procoagulative DAMPs into circulation will be important to further understand the pathophysiology of MOF. Not only does this have the potential to impact diagnostic decision-making early in the course of injury but may also be incorporated as a therapeutic target in upstream treatment and better yet, prevention of MOF.

\section{Methods}

\section{Cell culture and reagents}

Sprague-Dawley rat normal skin fibroblasts (ATCC) were maintained in Eagle's minimum essential medium supplemented with $10 \%$ fetal bovine serum (FBS), $1 \times$ nonessential amino acids and $1 \mathrm{mM}$ sodium pyruvate (all from Invitrogen). Mouse melanoma cell line B16-F0 (ATCC), mouse pancreatic cancer cell line PANC-02 (kindly provided by Rebekah White, University of California, San Diego, California, USA), and mouse macrophage cell line RAW264.7 (ATCC) were maintained in Dulbecco's modification of Eagle medium (DMEM) supplemented with 10\% FBS. TLR reporter cell lines, including HEK-hTLR2, HEK-hTLR3, HEK-hTLR4, and HEK-hTLR9 cells (all from InvivoGen), stably express genes encoding transcription factor NF- $\mathrm{KB}$-inducible secreted embryonic alkaline phosphatase (SEAP) and corresponding TLR, and these reporter cells were maintained by following the manufacturer's instructions. All cells were incubated at $37^{\circ} \mathrm{C}$ in a humidified atmosphere with $5 \% \mathrm{CO}_{2}$. To determine $\mathrm{TF}$ expression, cells were stained with phycoerythrin-conjugated (PE-conjugated) anti-mouse CD142 antibody (FAB3178P, R\&D Systems) or goat IgG PE-conjugated isotype antibody (IC108P, R\&D Systems).

\section{Preparation of DAMPs and PAMPs}

DAMPs and PAMPs were prepared by ex vivo breakage of cells and bacteria, respectively. Fully confluent fibroblasts, B16-F0, and PANC-02 cells in a T175 flask and Pseudomonas aeruginosa (PAO1, ATCC 15692) in 100 $\mathrm{mL}$ of LB broth (ATCC) were harvested, followed by resuspension in $5 \mathrm{~mL}$ of Dulbecco's phosphate-buffered saline (DPBS) (MilliporeSigma). The cells and bacteria were sonicated for 1.5 minutes with a Branson Ultrasonics Sonifier 250, followed by centrifugation for 5 minutes at $300 \mathrm{~g}$ and 1,000 $\mathrm{g}$. Pellets containing live cells, cell debris, and large organelles were discarded, and supernatants containing DAMPs (or PAMPs), microparticles, and extracellular vesicles were harvested. The amounts of total DAMPs and PAMPs in the supernatants were quantified using a BCA protein assay kit (Thermo Fisher Scientific). The levels of specific DAMPs, such as exDNAs and HMGB1, were measured using Quant-iT PicoGreen DNA assay kit (Thermo Fisher Scientific) and HMGB1 enzyme-linked immunosorbent assay (ELISA) kit (Tecan), respectively, by following the manufacturer's instructions. Gram-negative bacteria outer membrane LPS (026:B6, Sigma-Aldrich), bovine kidney HS (Sigma-Aldrich), and endotoxin-free disulfide HMGB1 (Tecan) were used as a representative PAMP and DAMP.

\section{In vitro innate immune stimulation with DAMPs and PAMPs}

TLR reporter cells were stimulated with DAMPs or PAMPs in a 96-well plate. Upon binding to their cognate ligands, TLR signaling activates NF- $\mathrm{kB}$ that leads to expression and release SEAP from the TLR reporter cells. The level of NF- $\mathrm{BB}$ activity was measured by quantification of SEAP in culture media using a colorimetric assay, as described previously (39). To stimulate mouse macrophages, RAW264.7 cells were incubated overnight with DAMPs or PAMPs. TNF- $\alpha$ and IL-10 production by RAW264.7 cells was determined using BD OptEIA ELISA sets (BD Biosciences).

\section{Plasma coagulation assay}

Plasma coagulation assay was performed by described previously (39). Briefly, $50 \mu \mathrm{L}$ normal pooled mouse plasma in sodium citrate (C57BL/6) (Biochemed Services) or $50 \mu \mathrm{L}$ normal pooled human plasma in sodium citrate (George King Bio-Medical Inc.) was incubated for 5 minutes with $5 \mu \mathrm{L}$ DAMPs or PAMPs at $37^{\circ} \mathrm{C}$, followed by the addition of $\mathrm{CaCl}_{2}(25 \mathrm{mM})$. Coagulation activator silica (Stago) was used as a positive control. Clotting times were recorded using STart Hemostasis Analyzer (Diagnostica Stago). 
Separation of large and small particles and soluble DAMPs

Particles and soluble DAMPs were isolated from cell culture supernatants or plasma samples by differential centrifugation, as described previously (40). Briefly, the culture supernatants and plasma samples were centrifuged for 5 minutes at $300 \mathrm{~g}$ and 3,000 $\mathrm{g}$ to remove live cells, large cell debris, and organelles. Pellets were discarded, and supernatants were further centrifuged for 1 hour at $20,000 \mathrm{~g}$ and $4^{\circ} \mathrm{C}$ to obtain large particle pellet, followed by centrifugation for 1 hour at $100,000 \mathrm{~g}$ and $4^{\circ} \mathrm{C}$ to obtain small particle pellet. The supernatant after 100,000 $\mathrm{g}$ centrifugation was used as a soluble DAMP-containing fraction. The amounts of particles and soluble fraction were quantified using the BCA protein assay.

In vivo induction of MOF and mortality by DAMPs and PAMPs

Mouse DAMPs or PAMPs in DPBS were administered 1 to 5 times either intraperitoneally or i.v.. At 2, 24, 48, and 168 hours after the first injection, $100 \mu \mathrm{L}$ to $200 \mu \mathrm{L}$ of blood was collected in $1.5-\mathrm{mL}$ Eppendorf tubes coated with ethylenediaminetetraacetic acid (EDTA), followed by centrifugation for 15 minutes at $1,500 \mathrm{~g}$ and $4^{\circ} \mathrm{C}$ to obtain plasma. Plasma levels of TNF- $\alpha$ and IL- 6 were determined using BD OptEIA ELISA sets. The levels of liver and kidney injury were determined by measuring plasma ALT and creatinine using ALT activity assay and creatinine assay kits, respectively (both from MilliporeSigma). TATc level was measured using mouse TATc ELISA kit (Abcam). The amount of plasma exDNA was quantified using a Quant-iT PicoGreen DNA assay kit.

\section{Cell growth, cell death, and cell cycle analyses}

Cell growth rate was measured using CellTiter 96 cell proliferation assay kit (Promega), according to the manufacturer's instructions. Cell death was measured using either trypan blue staining (Thermo Fisher Scientific) or PE annexin V apoptosis detection kit (BD Biosciences). Cell cycle status was determined using flow cytometry after staining with propidium iodide (PI).

\section{Statistics}

The statistical significance of results between groups was determined using the paired $t$ test, Mann-Whitney test, Kruskal-Wallis test, and Dunnett's or Tukey's multiple-comparisons test. Significance of survival was determined by log-rank (Mantel-Cox) test. A probability of less than $0.05(P<0.05)$ was used for statistical significance.

\section{Study approval}

Animals. C57BL/6 mice (Jackson Laboratory) were housed in a specific pathogen-free barrier facility and used at 6 to 12 weeks of age. All experimental procedures involving the use of mice were performed in accordance with the guidelines and in compliance with the Animal Care and Use Committee of Duke University.

Human blood samples. All human plasma and serum samples from sodium citrated blood were obtained according to a protocol approved by the Institutional Review Board of Duke University Medical Center. Patients with injury or illness requiring surgical care or treatment in a critical care or emergency setting older than age 18 years were included. Patient samples were obtained on or near admission date and throughout the clinical course of the patient.

\section{Author contributions}

Acquisition of data were done by JE, JK, US, and JL. The study conception and design were done by JE and $\mathrm{JL}$. The data interpretation and manuscript preparation were done by JE, MK, ATL, AB, ADK, JL, EAE.

\section{Acknowledgments}

We thank David S. Pisetsky and Diane Spencer for providing an ultracentrifuge. We thank Sara Miller and Ricardo Vancini with the Duke Electron Microscopy Shared Resource in their assistance with this study. This study was partially supported by the DoD/SC2i initiative: Department of Defense Health Program Joint Program Committee 6/Combat Casualty Care (USUHS HT9404-13-1-0032 and USUHS HU000115-2-0001) (ADK, EAE). We also thank Chandra Almond and the rest of the research coordinators of the Surgical Critical Care Initiative that assisted in patient sample collection.

Address correspondence to: Jaewoo Lee, PO Box 103035, Duke University Medical Center, Durham, North Carolina 27710, USA. Phone: 919.613.5045; Email: jaewoo.lee@duke.edu. 
1. Dewar DC, Tarrant SM, King KL, Balogh ZJ. Changes in the epidemiology and prediction of multiple-organ failure after injury. J Trauma Acute Care Surg. 2013;74(3):774-779.

2. Sauaia A, et al. Temporal trends of postinjury multiple-organ failure: still resource intensive, morbid, and lethal. J Trauma Acute Care Surg. 2014;76(3):582-593.

3. Prin M, Li G. Complications and in-hospital mortality in trauma patients treated in intensive care units in the United States, 2013. Inj Epidemiol. 2016;3(1):18.

4. Singer M, et al. The Third International Consensus Definitions for Sepsis and Septic Shock (Sepsis-3). JAMA. 2016;315(8):801-810.

5. Osterbur K, Mann FA, Kuroki K, DeClue A. Multiple organ dysfunction syndrome in humans and animals. J Vet Intern Med. 2014;28(4):1141-1151.

6. Opal SM, et al. Relationship between plasma levels of lipopolysaccharide (LPS) and LPS-binding protein in patients with severe sepsis and septic shock. J Infect Dis. 1999;180(5):1584-1589.

7. Simmons JD, et al. Elevated levels of plasma mitochondrial DNA DAMPs are linked to clinical outcome in severely injured human subjects. Ann Surg. 2013;258(4):591-598.

8. Di Caro V, et al. Plasma mitochondrial DNA--a novel DAMP in pediatric sepsis. Shock. 2016;45(5):506-511.

9. Kawai C, et al. Circulating extracellular histones are clinically relevant mediators of multiple organ injury. Am J Pathol. 2016;186(4):829-843

10. Darrabie MD, et al. Toll-like receptor activation as a biomarker in traumatically injured patients. J Surg Res. 2018;231:270-277.

11. Takeuchi O, Akira S. Pattern recognition receptors and inflammation. Cell. 2010;140(6):805-820.

12. Delano MJ, Ward PA. The immune system's role in sepsis progression, resolution, and long-term outcome. Immunol Rev. 2016;274(1):330-353.

13. Rittirsch D, Flierl MA, Ward PA. Harmful molecular mechanisms in sepsis. Nat Rev Immunol. 2008;8(10):776-787.

14. Gando S, Nanzaki S, Kemmotsu O. Disseminated intravascular coagulation and sustained systemic inflammatory response syndrome predict organ dysfunctions after trauma: application of clinical decision analysis. Ann Surg. 1999;229(1):121-127.

15. Murakami H, et al. Disseminated intravascular coagulation (DIC) at an early phase of trauma continuously proceeds to DIC at a late phase of trauma. Clin Appl Thromb Hemost. 2012;18(4):364-369.

16. Kushimoto S, et al. Clinical course and outcome of disseminated intravascular coagulation diagnosed by Japanese Association for Acute Medicine criteria. Comparison between sepsis and trauma. Thromb Haemost. 2008;100(6):1099-1105

17. Eppensteiner J, Davis RP, Barbas AS, Kwun J, Lee J. Immunothrombotic activity of damage-associated molecular patterns and extracellular vesicles in secondary organ failure induced by trauma and sterile insults. Front Immunol. 2018;9:190.

18. Smith SA, et al. Polyphosphate exerts differential effects on blood clotting, depending on polymer size. Blood. 2010;116(20):4353-4359.

19. Hutcheson JD, Schlicher RK, Hicks HK, Prausnitz MR. Saving cells from ultrasound-induced apoptosis: quantification of cell death and uptake following sonication and effects of targeted calcium chelation. Ultrasound Med Biol. 2010;36(6):1008-1021.

20. Cao X. Self-regulation and cross-regulation of pattern-recognition receptor signalling in health and disease. Nat Rev Immunol. 2016;16(1):35-50.

21. Delano MJ, Ward PA. Sepsis-induced immune dysfunction: can immune therapies reduce mortality? J Clin Invest. 2016;126(1):23-31.

22. Monguió-Tortajada M, Franquesa M, Sarrias MR, Borràs FE. Low doses of LPS exacerbate the inflammatory response and trigger death on TLR3-primed human monocytes. Cell Death Dis. 2018;9(5):499.

23. Vadiveloo PK, Keramidaris E, Morrison WA, Stewart AG. Lipopolysaccharide-induced cell cycle arrest in macrophages occurs independently of nitric oxide synthase II induction. Biochim Biophys Acta. 2001;1539(1-2):140-146.

24. Ning Y, Kim JK, Min HK, Ren S. Cholesterol metabolites alleviate injured liver function and decrease mortality in an LPS-induced mouse model. Metab Clin Exp. 2017;71:83-93.

25. Li Q, et al. Inhibition of autophagy with 3-methyladenine is protective in a lethal model of murine endotoxemia and polymicrobial sepsis. Innate Immun. 2018;24(4):231-239.

26. Rao LV, Pendurthi UR. Regulation of tissue factor coagulant activity on cell surfaces. J Thromb Haemost. 2012;10(11):2242-2253.

27. Fuchs TA, et al. Extracellular DNA traps promote thrombosis. Proc Natl Acad Sci USA. 2010;107(36):15880-15885.

28. Lord JM, et al. The systemic immune response to trauma: an overview of pathophysiology and treatment. Lancet. 2014;384(9952):1455-1465.

29. Mira JC, et al. The epidemiology of chronic critical illness after severe traumatic injury at two level-one trauma centers. Crit Care Med. 2017;45(12):1989-1996.

30. Frantzeskaki F, Armaganidis A, Orfanos SE. Immunothrombosis in acute respiratory distress syndrome: cross talks between inflammation and coagulation. Respiration. 2017;93(3):212-225

31. Keragala CB, Draxler DF, McQuilten ZK, Medcalf RL. Haemostasis and innate immunity - a complementary relationship: a review of the intricate relationship between coagulation and complement pathways. Br J Haematol. 2018;180(6):782-798.

32. Aneja RK, et al. Preconditioning with high mobility group box 1 (HMGB1) induces lipopolysaccharide (LPS) tolerance. $J$ Leu koc Biol. 2008;84(5):1326-1334.

33. Major JS, Welbourne J. Nosocomial infection in trauma intensive care. J Intensive Care Soc. 2015;16(3):193-198.

34. Fry DE. Sepsis, systemic inflammatory response, and multiple organ dysfunction: the mystery continues. Am Surg. 2012;78(1):1-8.

35. Engelmann B, Massberg S. Thrombosis as an intravascular effector of innate immunity. Nat Rev Immunol. 2013;13(1):34-45.

36. Ito T, et al. High-mobility group box 1 protein promotes development of microvascular thrombosis in rats. J Thromb Haemost. 2007;5(1):109-116.

37. Massberg S, et al. Reciprocal coupling of coagulation and innate immunity via neutrophil serine proteases. Nat Med. 2010;16(8):887-896. 
38. Müller I, et al. Intravascular tissue factor initiates coagulation via circulating microvesicles and platelets. FASEB J. 2003;17(3):476-478

39. Lee J, et al. Nucleic acid scavenging microfiber mesh inhibits trauma-induced inflammation and thrombosis. Biomaterials. 2017;120:94-102.

40. Aatonen MT, Ohman T, Nyman TA, Laitinen S, Grönholm M, Siljander PR. Isolation and characterization of platelet-derived extracellular vesicles. J Extracell Vesicles. 2014;3:10.3402/jev.v3.24692. 